\title{
Synthesis of PEG and quaternary ammonium grafted silicone copolymers as nanoemulsifiers
}

Srishti Gupta ${ }^{1}$, Pummy Singh ${ }^{1}$, Babak Moghadas ${ }^{2}$, Bradley J. Grim ${ }^{1}$, Vikram D. Kodibagkar ${ }^{2}$, and Matthew D. Green ${ }^{1, *}$

${ }^{1}$ Department of Chemical Engineering; School for Engineering of Matter, Transport and Energy, Arizona State University, Tempe, AZ 85287

${ }^{2}$ School of Biological and Health Systems Engineering, Arizona State University, Tempe, AZ 85287

*Corresponding author: Matthew Green (Email: mdgreen8@asu.edu)

\section{Supporting Information}

Table S1. Representative average Bond numbers for PEG and ammonium grafted silicone copolymers at solution concentrations of $\sim 1.0 \mathrm{mM}$ in water.

\begin{tabular}{|c|c|}
\hline Sample & Bond Number \\
\hline PHMS- $g$-PEG $(3)_{20}$ & 0.30 \\
\hline PHMS-g-PEG(3) $)_{15}-g$-QUAT 5 & 0.29 \\
\hline PHMS- $g$-PEG $(3)_{10}-g$-QUAT 10 & 0.26 \\
\hline PHMS- $g$-PEG(11) $)_{20}$ & 0.27 \\
\hline PHMS-g-PEG(11) $)_{15}-g$-QUAT $T_{5}$ & 0.26 \\
\hline PHMS- $g$-PEG(11) $)_{10}-g$-QUAT 10 & 0.28 \\
\hline
\end{tabular}




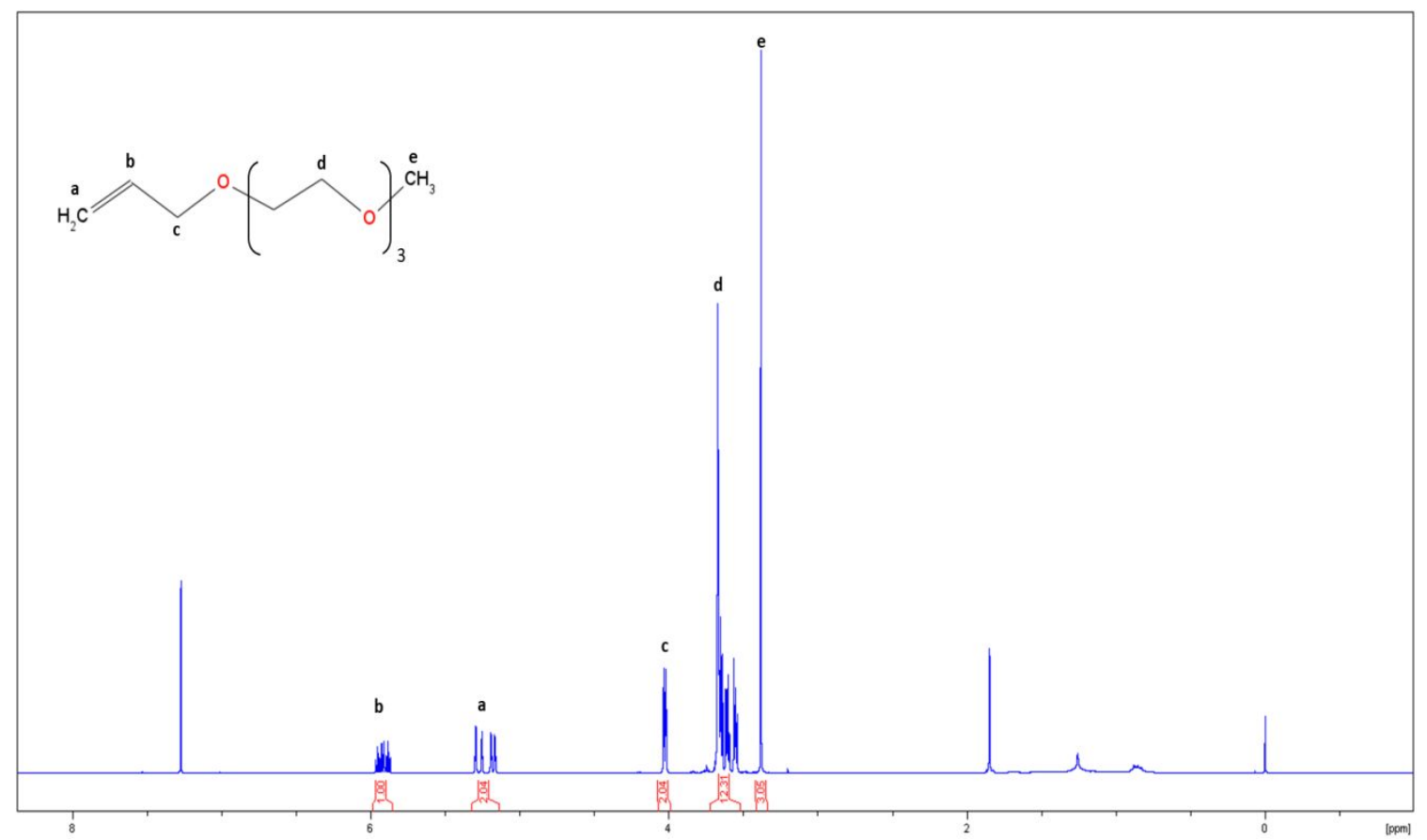

Figure S1: Allyl poly(ethylene glycol) mono methyl ether: ${ }^{1} \mathrm{H}$ NMR $\left(\mathrm{CDCl}_{3}, 400 \mathrm{MHz}\right): \delta 5.85$ $(\mathrm{m}, 1 \mathrm{H}), 5.15(\mathrm{dd}, 2 \mathrm{H}), 3.95(\mathrm{~d}, 2 \mathrm{H}), 3.45-3.65(\mathrm{~m}), 3.35(\mathrm{~s}, 3 \mathrm{H})$.

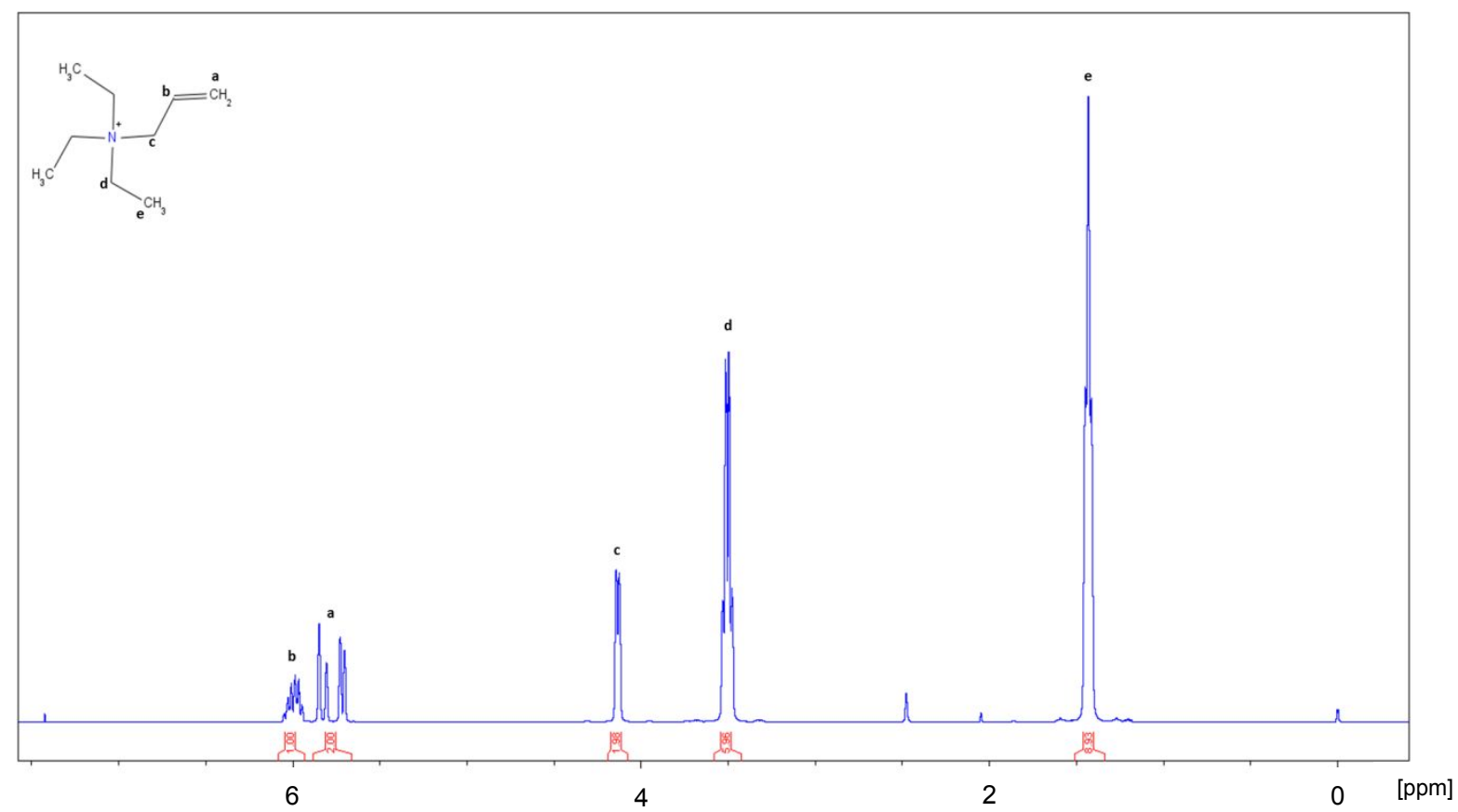

Figure S2: Allyl triethylammonium bromide: ${ }^{1} \mathrm{H} \mathrm{NMR}\left(\mathrm{CDCl}_{3}, 400 \mathrm{MHz}\right): \delta 6.0(\mathrm{~m}), 5.8(\mathrm{dd}$, $2 \mathrm{H}), 4.15$ (d, 2H), 3.45 (q, 6H), $1.4(\mathrm{t}, 9 \mathrm{H})$. 


\section{Supporting Information}

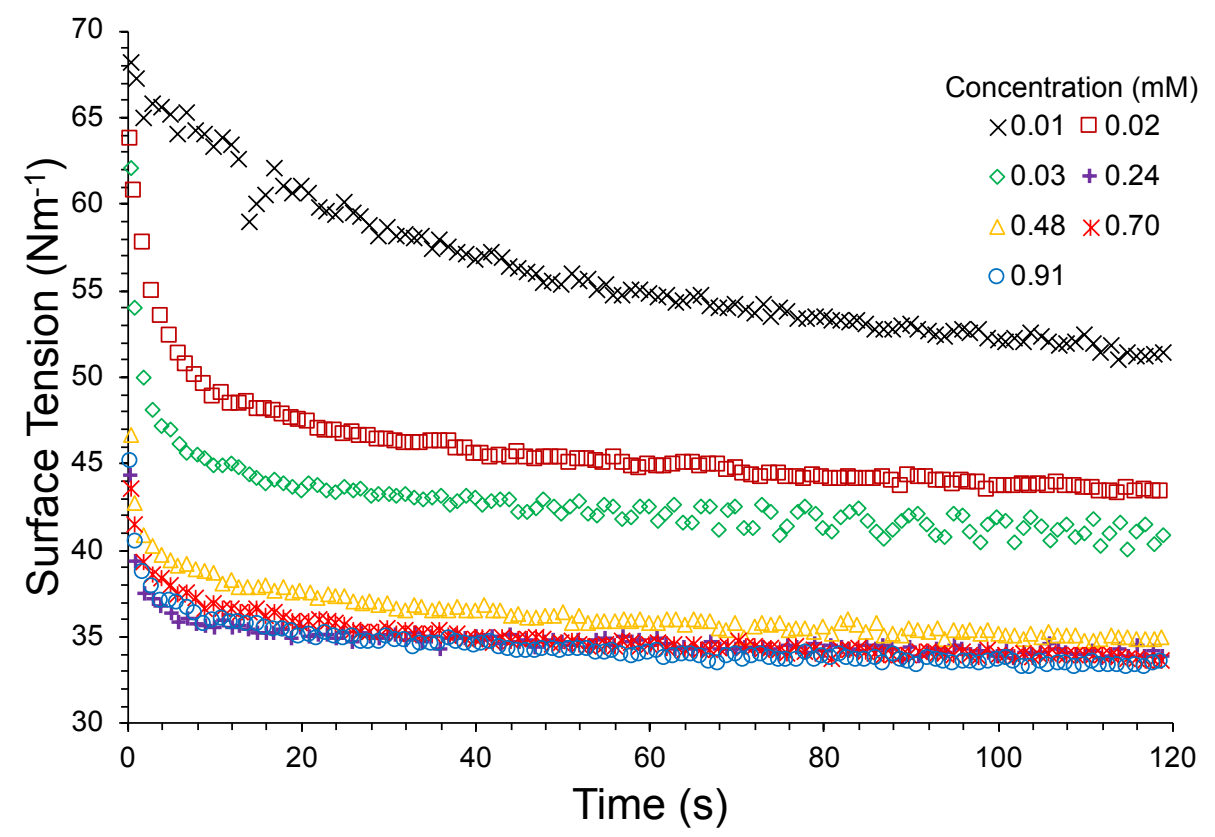

Figure S3. A representative plot of surface tension versus time for several solutions of PHMS- $g$ $\mathrm{PEG}(11)_{15}-\mathrm{g}-\mathrm{QUAT} \mathrm{T}_{5}$ in water. Measurements of surface tension were taken after $80 \mathrm{~s}$, typically between $100-120 \mathrm{~s}$.

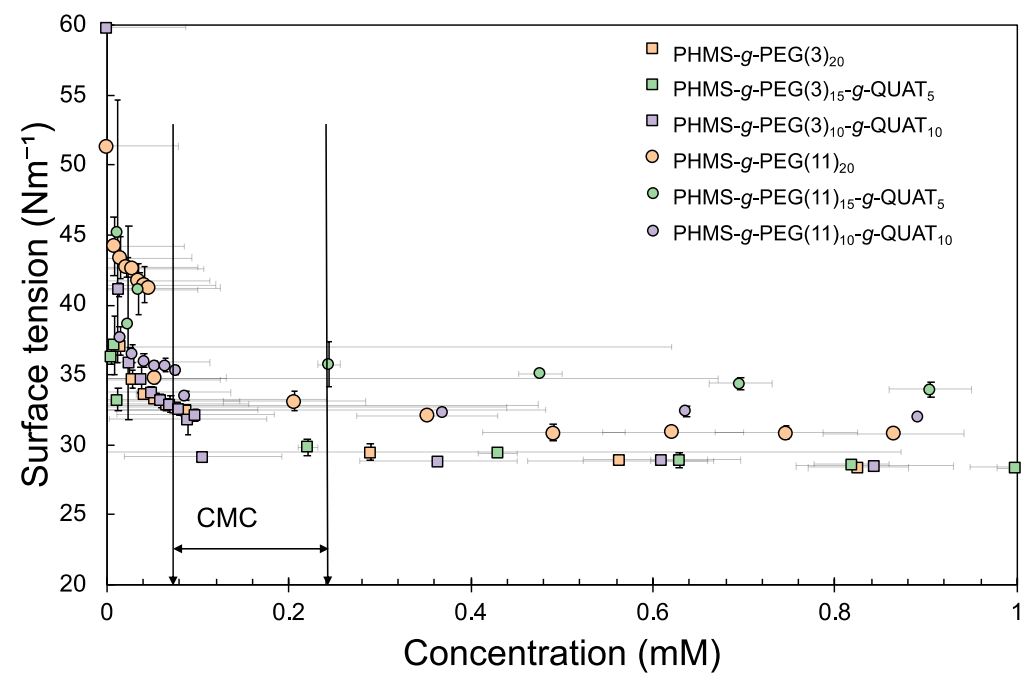

Figure S4. Dependence of surface tension on polymer concentration for the PEG and ammonium grafted siloxane copolymers. Using a linear axis for concentrations allows for the observation that surface tension remains constant above the reported CMC. 


\section{Supporting Information}

Table S2. Hydrodynamic diameter results obtained via the intensity distribution profiles from dynamic light scattering for PEG and ammonium grafted silicone copolymers (PHMS-g-PEG(n) $\left.)_{x}-g-Q U A T_{y}\right)$.

\begin{tabular}{ccc}
\hline & $\begin{array}{c}\text { Hydrodynamic diameter } \\
(\mathrm{nm})\end{array}$ & PDI \\
\hline PHMS- $g$-PEG(3) & $163.10 \pm 10.89$ & $0.82 \pm 0.08$ \\
${\text { PHMS- } g \text {-PEG(3) }(3)_{15}-g \text {-QUAT }}_{5}$ & $167.80 \pm 1.27$ & $0.26 \pm 0.01$ \\
${\text { PHMS- } g \text {-PEG }(3)_{10}-g \text {-QUAT }}_{10}$ & $172.40 \pm 4.67$ & $0.76 \pm 0.04$ \\
\hline PHMS- $g$-PEG $(11)_{20}$ & $588.25 \pm 46.46$ & $0.83 \pm 0.14$ \\
${\text { PHMS- } g \text {-PEG }(11)_{15}-g \text {-QUAT }}_{5}$ & $436.85 \pm 35.00$ & $0.48 \pm 0.03$ \\
${\text { PHMS- } g \text {-PEG }(11)_{10}-g-Q U A T_{10}}$ & $214.90 \pm 8.63$ & $0.33 \pm 0.13$ \\
\hline
\end{tabular}

Table S3. Hydrodynamic diameter results obtained via the number distribution profiles from DLS for PEG and ammonium grafted silicone copolymers (PHMS- $g-\mathrm{PEG}(\mathrm{n})_{\mathrm{x}}-\mathrm{g}-\mathrm{QUAT} \mathrm{T}_{\mathrm{y}}$ ).

\begin{tabular}{|c|c|c|c|}
\hline & \multicolumn{2}{|c|}{ Hydrodynamic diameter (nm) } & \multirow[t]{2}{*}{ PDI } \\
\hline & Peak 1 & Peak 2 & \\
\hline PHMS- $g-\mathrm{PEG}(3)_{20}$ & $8.89 \pm 2.1$ & $38.53 \pm 14.08$ & $0.82 \pm 0.08$ \\
\hline PHMS-g-PEG(3) $)_{15}-g-\mathrm{QUAT}_{5}$ & $55.89 \pm 18.45$ & $180.1 \pm 64.21$ & $0.26 \pm 0.01$ \\
\hline PHMS-g-PEG(3) ${ }_{10}-g-$ QUAT $_{10}$ & $28.52 \pm 7.78$ & $445 \pm 72.41$ & $0.76 \pm 0.04$ \\
\hline PHMS- $g$-PEG $(11)_{20}$ & $11.47 \pm 6.74$ & $171.6 \pm 11.17$ & $0.83 \pm 0.14$ \\
\hline PHMS-g-PEG(11) $)_{15}-g-$ QUAT $_{5}$ & $33.62 \pm 5.65$ & $256.5 \pm 28.07$ & $0.48 \pm 0.03$ \\
\hline PHMS- $g$-PEG(11) $)_{10}-g$-QUAT 10 & & $236.1 \pm 27.58$ & $0.33 \pm 0.13$ \\
\hline
\end{tabular}


Supporting Information
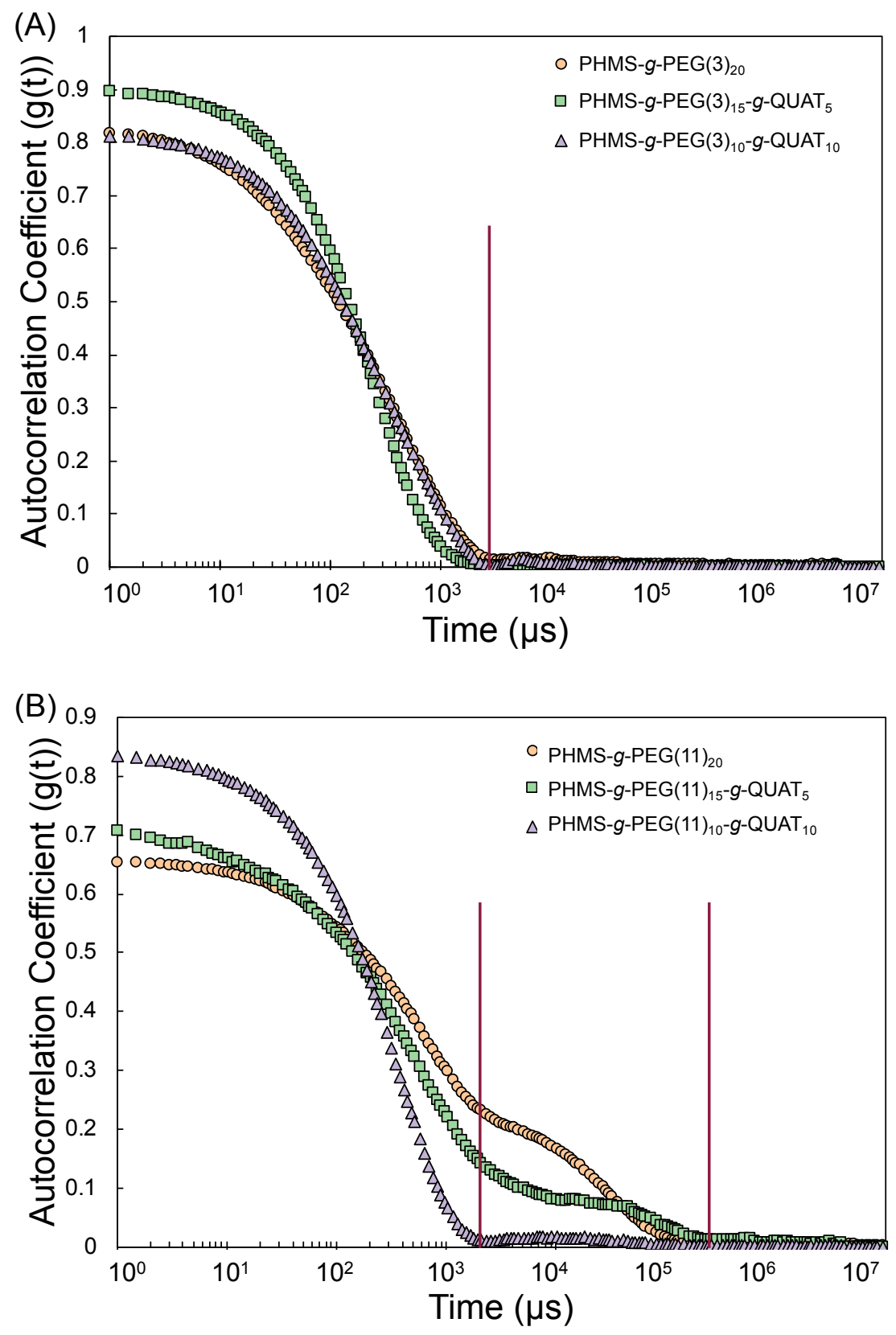

Figure S5. Autocorrelation coefficient data obtained via DLS for (a) PEG(3) and (b) PEG(11) grafted silicone copolymers (PHMS- $g-\mathrm{PEG}(\mathrm{n})_{\mathrm{x}}-\mathrm{g}-\mathrm{QUAT}_{\mathrm{y}}$ ) at $25^{\circ} \mathrm{C}$ in water. 


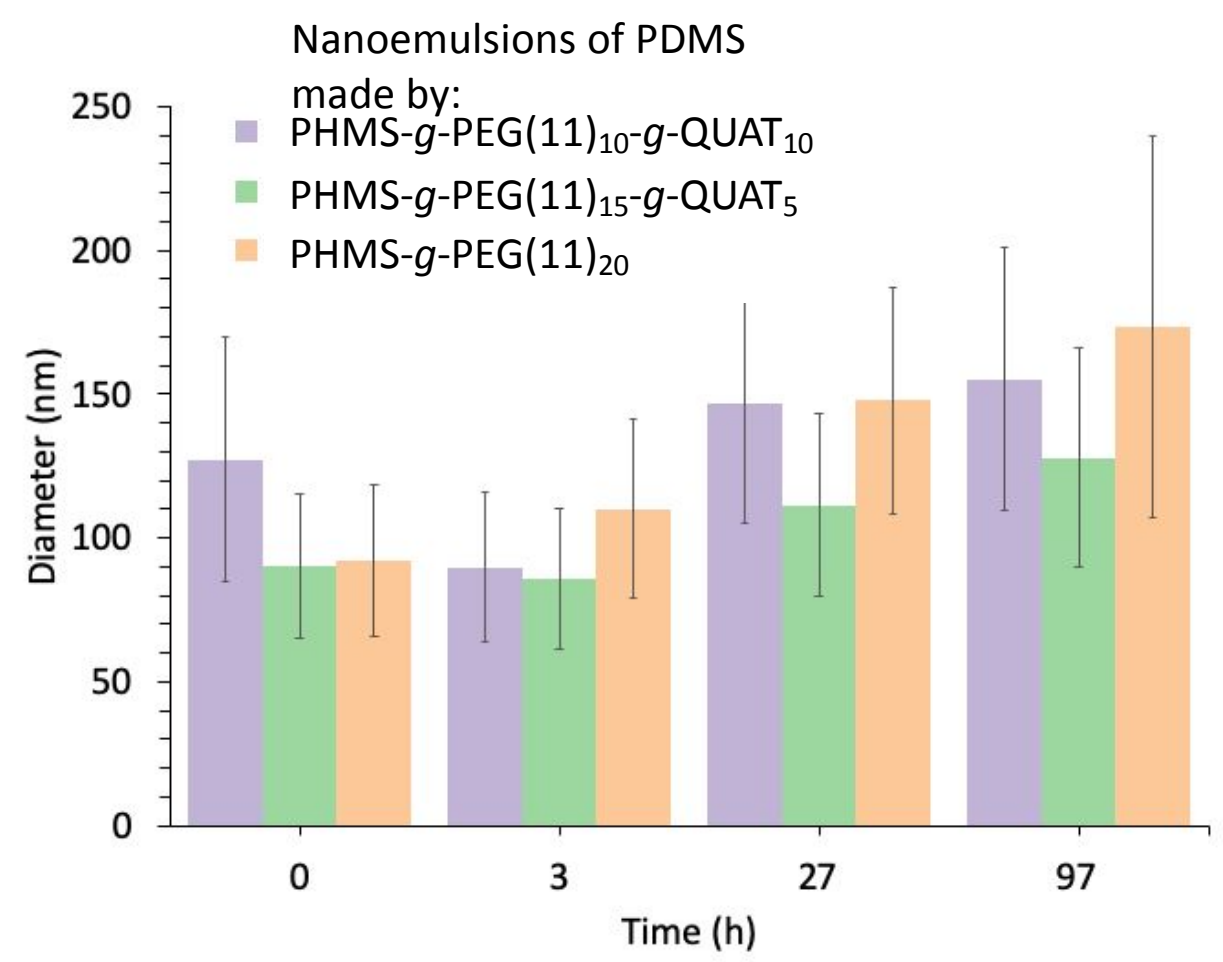

Figure S6. Dynamic light scattering (DLS) data for particle size estimation of nanoemulsions from number distribution profiles, wherein polydimethylsiloxane $\left(M_{n}=410 \mathrm{~g} / \mathrm{mol}\right)$ was emulsified using

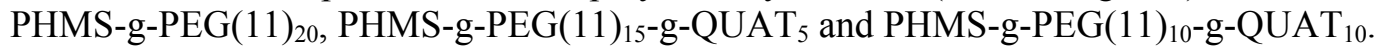
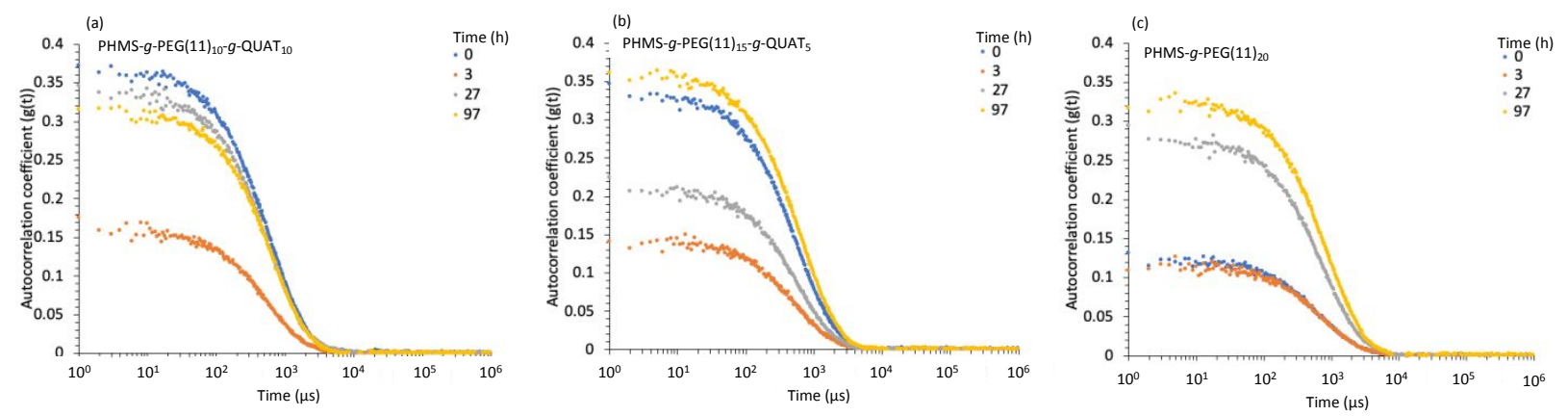

Figure S7. Autocorrelation data obtained from DLS for nanoemulsions of PDMS made by (a) PHMS- $g$ PEG(11) $)_{10}-g$-QUAT 10 , (b) PHMS- $g$-PEG(11) $)_{15}-g-\mathrm{QUAT}_{5}$, and (c) PHMS- $g$-PEG(11) $)_{20}$ PEG and ammonium grafted silicone copolymers at $25^{\circ} \mathrm{C}$ in water. 